\title{
Markov Model-based Handwritten Signature Verification
}

\author{
Alan McCabe and Jarrod Trevathan \\ School of Mathematics, Physics and Information Technology, James Cook University, Australia \\ Email: alan@mymait.com, jarrod.trevathan@jcu.edu.au
}

\begin{abstract}
Biometric security devices are now permeating all facets of modern society. All manner of items including passports, driver's licences and laptops now incorporate some form of biometric data and/or authentication device. As handwritten signatures have long been considered the most natural method of verifying one's identity, it makes sense that pervasive computing environments try to capitalise on the use of automated Handwritten Signature Verification systems (HSV). This paper presents a HSV system that is based on a Hidden Markov Model (HMM) approach to representing and verifying the hand signature data. HMMs are naturally suited to modelling flowing entities such as signatures and speech. The resulting HSV system performs reasonably well with an overall error rate of $3.5 \%$ being reported in the best case experimental analysis.
\end{abstract}

\section{INTRODUCTION}

Handwritten Signature Verification (HSV) is an automated method of verifying a person by examining the features inherent in his/her handwritten signature. HSV systems can use both static and dynamic features to verify a signature. Static features encompass the physical properties of a signature such as shape, slant and size. Whereas dynamic features are derived from the characteristics of the way in which a person signs his/her name in real time (such as velocity, pressure, penup time, etc.). The goal for HSV is to reduce both the False Acceptance Rate (FAR) - the number of forgeries accepted as genuine; and the False Rejection Rate (FRR) - the number of genuine signature rejected as forgeries.

McCabe et al [10] describe a HSV system that uses a Neural Network (NN) machine learning technique to model and verify the features in a sample handwritten signature. The $\mathrm{NN}$ approach described is based on global features - that is, features that were extracted from the signature as a whole. No attempt was made to compare patterns that may exhibit temporal variations, that is, features that are local in nature.

Although the standalone system described in McCabe et al [10] performed quite well, it is clear that there is still a large amount of local information that is not being captured. This paper describes another system that focuses on characterising that local information through the use of local features and a statistical signal modelling technique known as a Hidden Markov Model (HMM).

HMMs (also referred to as "Markov sources" or "probabilistic functions of Markov chains" [13]) are finite stochastic automata, with a powerful capability of modelling time-varying dynamic patterns. Researchers have been using them for various modelling problems since as early as 1975 [1], particularly in speech recognition tasks [7], [13]. More recently they have undergone a popularity increase in handwriting analysis tasks including character recognition [3], [16] and HSV [4], [8].

Training HMMs is done in a fashion similar to the training of NNs in the sense that a feature set is extracted from the signature and a series of these sets is presented to the HMM. The HMM then tries to model the input data in some way. When testing a new signature (in signature verification tasks), instead of outputting a classification, the HMM outputs a probability indicating the likelihood that the test signature was produced by a given signer (or more specifically, the likelihood that the signature features could have been generated by the given HMM).

HMMs are naturally suited to modelling "flowing" entities such as speech and handwriting. They are made up of a series of states with transitions between these states. Signatures can be split up into different sections through a process of segmentation, with a similar number of sections to the number of states in the HMM. It is then possible to progress through the states of the HMM in a corresponding fashion to progressing through the segments of the signature. At each state, the local features of the signature (such as the average velocity, acceleration or duration in the current segment) are examined. Local features therefore can be naturally modelled by HMMs.

The HSV system described in this paper takes into account historical work performed in biometric verification using HMMs. It extracts several local features (both static and dynamic) from the signature data and models these using the best aspects of historical HMM modelling for HSV. The resulting system is tested on an experimental dataset that contains multiple samples of handwritten signatures (including both genuine and forgeries). The resulting system performs reasonably well with an Overall Error Rate (OER) of $3.5 \%$ (OER is equal to FAR + FRR).

This paper is organised as follows: Section II discusses the various design aspects of HMMs in relation to HSV. Section III describes the feature set used in the proposed HSV system. Section IV presents the experimentation conducted and results obtained in the development of the HMM-based HSV system. Section $\mathrm{V}$ provides some concluding remarks.

\section{PREVIOUS WORK}

HSV is still a relatively new application area for HMMs, with few truly intensive investigations being reported in the 
literature. Some of the more landmark studies with HMMs in HSV are presented here.

The basic approach to HMMs involves performing stochastic matching of a model and a signature using a sequence of probability distributions of the features along the signature [6]. The signing process is usually modelled with several states that constitute a Markov chain, each of them corresponding to a signature segment. The states are generally not directly observable (i.e., they are hidden) and only the signature's local features (such as tangent angles) can be observed.

A system that combined local and global information in multiple models is presented in [6]. Methods of combination such as the one described are of significant interest to this research and this particular approach is further discussed in future work. The local feature comparison used in the work though is of interest here and involves using HMMs to compare feature vectors. Six sample signatures were used to train the HMM, which modelled the user's signature as a series of states with probabilistic transitions between them. Given a test signature, the Viterbi algorithm [17], [13] is used to search for the most likely state sequence corresponding to the given observation sequence and give the accumulated likelihood score along that best path. The difference between this score and the mean likelihood obtained during training is then used as an error measure to classify a test signature as valid or a forgery. This approach resulted in an Equal Error Rate (EER) of around 5\% and the combination techniques (discussed later) reduce this to an error rate of $2.5 \%$ EER. (EER is the point at which the FAR and FRR are of equal value.)

Another early study into HMMs for HSV is reported in [18], which uses absolute angular direction along the trajectory of the signature, encoded as a sequence of angles. Each signature is divided into a fixed number of segments in order to obtain sequences of the same length, and the normalised angles then quantised into sixteen levels. Experiments are conducted using several model structures including left-to-right and parallel models. Training is done using the forward-backward algorithm and probabilities estimated using the Baum-Welch algorithm. Thirty-one writers contributed sixteen signatures each for use in evaluation in which eight signatures are used for training and eight used in testing (no skilled forgeries were used). The left-to-right model with arbitrary state skips is found to result in the lowest error rates. Additionally, the study found that increasing the number of states and decreasing the observation length leads to a decrease in the false rejections and an increase in the false acceptances (presumably because less detail is captured when the observation length is decreased). The best results reported are a $4.4 \%$ FAR and $1.75 \%$ FRR, although no skilled forgeries are used (only zeroeffort).

The MM engine forming part of the HMM described in this paper appears in related work in [8]. In this study, experimentation is performed using simple directional features for verification of handwritten passwords. The approach is based on segmenting the signature into conceptually significant parts (i.e., extraction of strokes) and then extracting a single directional feature from the stroke. The net displacement direction of each stroke is characterised as one of $n$ discrete directions and represented as a single integer (one integer value per stroke). The result of this processing is that a signature can be represented as a series of integer labels that characterises the basic directional changes undergone in the handwriting stream. This representation is invariant under scaling and the slight rotation that may occur in signature production. In the work, the optimal value for $n$ was found to be four (corresponding to quadrants in a Cartesian grid) and the signatures are modelled using a five-state MM (one state for each direction, plus a "pen-up" state). The work also attempts to incorporate the duration of a stroke by repeating the integer symbol every twenty-fifth of a second that the stoke was maintained. A particularly long strong in direction " 1 " for example results in a series of 1 's being placed into the integer string. The approach therefore records the handwriting's basic shape as well as incorporating some sense of timing and velocity.

Five signature samples are used as the reference set to train the model, which involved re-estimation of the state transition probabilities based on the five extracted integer strings. For each test sample (both genuine and forgeries) corresponding to the current writer, the forward algorithm is used to find the probability that the test writing came from the same individual as the reference writings. The test signature is only accepted as genuine if this probability is higher than a pre-determined threshold. The approach is tested on a database consisting of 720 genuine handwritten passwords from forty-seven writers, and 265 forgeries. The EER found is $11.25 \%$ for skilled forgeries and $0.64 \%$ for zero-effort forgeries (when forgers hadn't seen a password sample). The error rate needs to be made much lower for the system to be of any significant value, although results remain interesting because of the simplicity of the features used.

An examination of HMMs for HSV is presented in [4]. A formal definition of the HSV problem using HMMs was presented as: given an individual $i$, their signature is described with a model $\lambda_{i}$, a threshold $T_{i}$, and a sequence of input items $X=x_{1}, x_{2}, \ldots, x_{n}$, accept the signature if the condition $-\log \left(P\left(\lambda_{i} \mid X\right)\right) \leq T_{i}$ holds. The approach used involved segmenting the signature input into strokes based on zero crossings in the $y$ velocity. A feature vector of thirty-two components (a mix of spatial, dynamic and contextual features) is then extracted for each segment, including angular features and seldom-used pen-tilt angles in the $x$ and $y$ direction.

Each signature was modelled by a single left-to-right HMM with loop, forward and skip transitions with probabilities reestimated during training. The number of states in the model was set to 0.8 times the average number of feature vectors (i.e., strokes) per signature in the training data. The value of 0.8 was arrived at through trial-and-error experimentation and similar values for the number of states are reported elsewhere in the literature [5].

An extensive database of almost 5,000 signatures is used including genuine signatures, home-improved forgeries, over- 
the-shoulder forgeries and forgeries produced by forensic document examiners. Reported error rates include $2.58 \%$ and $1.11 \%$ FAR for over-the-shoulder forgeries and homeimproved forgeries respectively (this corresponds to an EER of $1.9 \%$ using the same threshold). Experiments conducted with "professional" forgeries produced by forensic document examiners provided with paper copies of the genuine signatures did not result in any degradation of these error rates. The speculated justification for this was that the professional forgers were not able to observe the dynamics of the original signers and were less focused on forging dynamics than forging signature shape.

This study also describes linear discriminant analysis performed in examining the discriminative value of the features. Features are ranked for each writer and these individual rankings combined to provide an overall perspective. In general, dynamic features are found to result in much more accurate verification than spatial or static features. The five most discriminative individual features include velocity and pressure information, as well as both pen-tilt features.

A comparison of on-line and off-line HSV using HMMs is made in [14]. Seven different features are investigated in the on-line system for there discriminative capabilities including pressure, angular information, velocity, acceleration and the Fourier transform of the signal (although no global features are used in the study). The off-line system divides the signature image into a fixed number of squares of approximately ten pixels in diameter, with each column then represented as a vector. Few details are given as to the structure of the HMMs used, other than that they are discrete models. The Viterbi algorithm is used to compute the probability that the feature observation sequence extracted from the test signature was generated by the corresponding HMM (trained using the reference signatures for the same signer). The database consisted of fourteen writers, each contributing twenty signatures, and a total of sixty forgeries were available. Combining velocity, Fourier transforms, pressure and bitmap features (from the off-line system) yields the best on-line error rates of $1.0 \%$ equal error, with $1.9 \%$ the best EER obtained using the purely off-line system. This study supports the claim that on-line signatures are more information rich than off-line, and also that HMMs are capable of taking advantage of this extra information.

\section{Methodology}

This section presents the methodology followed for the development of the HMM portion of this project. The system described in this paper is a parameter-based dynamic system, and as such the explanation of methodology starts with the features used in the HMM and is followed by the experimental setup and the training process.

\section{A. Signature Segmentation}

The segmentation of handwriting at conceptually significant points (these segments are known in the literature as "strokes") is very important to many handwriting processing applications.

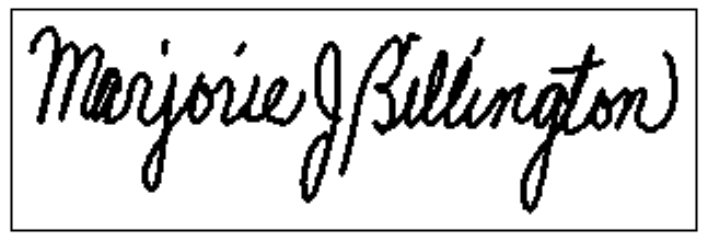

Fig. 1. Using the velocity based stroke technique for segmentation results in 290 strokes, whereas the extremum consistency approach results in 217 strokes.

Segmentation is of particular importance to this form of HSV where a HMM is used to model the individual segments. The traditional approach to this is typically to delimit the segments using minima in the pen-tip or the $y$ velocity.

A new approach to this segmentation, known as the $E x$ tremum Consistency (EC) method, is proposed as part of this research (see [9]). This method involves attempting to reduce the number of "false" minima occurring due to anomalies such as shaky hands, muscle fatigue or device/rounding errors etc. Figure 1 shows a typical signature from the signature database that contains 290 strokes according to the traditional Velocity Based Stroke (VBS) method. Using the EC approach, the number of strokes reduces to 217 .

McCabe and Trevathan [9] also presents the resulting improvement in error rates using the EC approach over other forms of segmentation and in one case the accuracy of a HSV system is reduced from $6.9 \%$ overall error (for a basic VBS implementation using gradient descent) to $2.3 \%$ overall error. There is a slight overhead associated with the EC calculations. However, the number of strokes extracted using this approach is typically reduced by around $25 \%$, so the overall signature processing procedure is generally faster.

\section{B. Extracted Features}

A discussion of features and feature extraction is presented in the corresponding section in McCabe et al [10]. Many of the HMM features are similar to those used as input to the NN (modified to apply to local strokes).

What follows now is a discussion of each of the features extracted for use in the HMM, as well as their significance and method of calculation. Each of these features is included in a feature array (often referred to as a feature vector) that acts as a single observation in the HMM.

Horizontal Length: The horizontal distance measured between the stroke's two most extreme points in the $x$ direction. See Figure 2 for an example.

Aspect Ratio: This is the ratio of the stroke length to the stroke height. This feature obviously remains invariant to scaling as if the user signs in a different size, both the length and the height will be altered proportionally and the aspect ratio will remain the same.

Curvature: This is a measure of how "flat" or how "curved" the stroke is. A high value for curvature means that the stroke is highly curved, which is associated with a turning point in the handwriting. (See Figure 3.) 


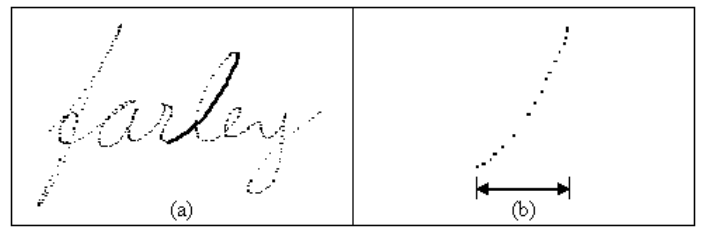

Fig. 2. This figure represents the horizontal length of a typical stroke. (a) contains the original handwritten sample with an extracted stroke in bold. (b) shows an expanded view of that same stroke with the horizontal length marked.

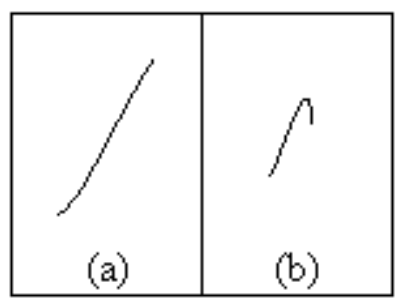

Fig. 3. Different strokes can result in quite different curvature values. For example, (a) shows a sample stroke that is quite flat, resulting in a curvature value of 0.01. Conversely (b) shows a sample with a much more pronounced curve that results in the higher curvature value of 0.40 .

Curvature is calculated as the ratio of the strokes path length to the overall length minus one. The path length is the sum of distances between each consecutive point in the sampled stroke. The overall length is the physical, or Euclidean, distance between the stroke start-point and the stroke end-point.

Maximum Velocity: This is a dynamic feature of the stroke meaning that it is very difficult for a potential forger to copy. In addition, velocity is a highly stable feature, easily repeatable by a genuine user [12]. The calculation of pen-tip velocity is done in terms of components velocities, $v_{x}$ and $v_{y}$, (calculated as the first derivative of the $x$ and $y$ streams):

$$
v=\sqrt{v_{x}^{2}+v_{y}^{2}}
$$

Average Velocity: The average velocity is a personal measure of the how fast the pen-tip is travelling across the surface of the tablet. This is calculated as the average of all velocity values extracted from the stroke.

Average Absolute Acceleration: This refers to the average absolute value of the acceleration and deceleration measurements. It is computed as the second derivative of the data stream (or the derivative of the velocity values calculated earlier). This feature captures the mean rate of change in velocity in both positive and negative directions.

Maximum Acceleration: While this feature is slightly less stable than some others, its dynamic nature and difficulty in forging still make it a useful characteristic.

Maximum Deceleration: This is a dynamic feature that measures the rate at which the pen-tip's velocity decreases as it approaches the end of a stroke.

Stroke Slant Using All Points: There are a number of techniques that measure various aspects of the slant (see [10]).

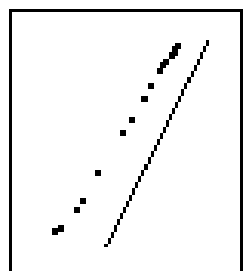

Fig. 4. Handwriting slant calculated using stroke end-points. This is the same stroke as shown in Figure 3, depicted here as the series of sampled points. The solid line to the immediate right is the calculated slant.

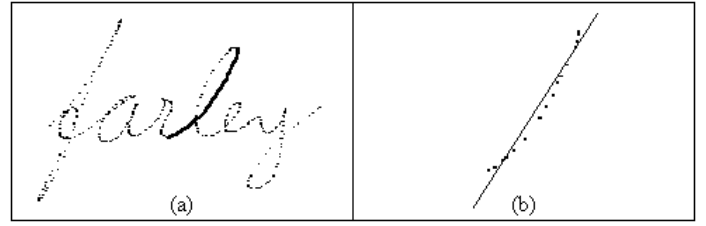

Fig. 5. An illustration of handwriting slant calculated through regression. (a) shows the original word as a series of sampled points with the extracted stroke in bold and (b) shows slant calculated via regression.

The main approach to calculating the stroke slant is to take the mean of all gradient values between the spatially resampled neighbouring points.

Handwriting Slant Using Stroke End-points: The gradient of the stroke is taken as simply the gradient between the stroke start-point and the stroke end-point. This was found to be less stable than the previous method for slant calculation and eventually removed from the feature set due to excessive variability. Figure 4 presents an example slant calculation using this method. This shows a calculated slant that does not seem to accurately match the slant of the stroke.

Handwriting Slant Through Regression: This approach involves performing linear regression using all the points in the stroke. The resulting line is taken as the gradient for the stroke and is illustrated in Figure 5.

Handwriting Slant Using Cai and Liu Technique: This method of determining slant is based on handwriting recognition techniques [2]. Calculation of this feature is done in an identical manner to the way described in McCabe et al [10].

Stroke Concavity: This is a measure of how far the stroke is from being a straight line. A stroke of high concavity does not closely follow the imaginary line drawn from the stroke's startpoint to the end-point. Calculation of the concavity value is done by firstly performing linear regression using the points in the stroke to obtain the line-of-best-fit. This feature then becomes a measure of how well the points in the stroke "fit" that line, or how well the points are approximated by that line. The second step is to apply the following formula for each stroke:

$$
\text { Stroke Concavity }=\sqrt{\sum_{i=1}^{n}\left(s_{i}-r_{i}\right)^{2}}
$$

where: 
- $n$ is the number of points in the stroke;

- $s_{i}$ is the $i^{t h}$ point in the stroke;

- $r_{i}$ is the coordinate along the line-of-best-fit that is the least distance from $s_{i}$.

Stroke Duration: This is a simple feature that records the amount of elapsed time between the first sampled point and the last sampled point in the extracted stroke. This feature remains quite stable between corresponding strokes and is not obvious to a potential forger.

Horizontal Velocity: This is the average absolute velocity in the $x$ direction. This measures how fast the pen-tip moves horizontally when producing the stroke and is related to pentip velocity, horizontal length and acceleration. It is impossible for a forger to discern the horizontal velocity from an off-line copy of the writing. This feature is calculated as the ratio of absolute horizontal distance to the duration in which the stroke was produced.

Mean Pen-Tip Pressure: This is a measure of the amount of vertical pressure being applied by the pen to the top of the tablet. This is an option available on most existing tablet and stylus hardware devices and is typically measured by an accurate sensor in the tip of the pen. Pressure, like many features used in this system, is very difficult for a forger to discern from an off-line copy of the handwriting. Although pen-tip pressure is less stable than other dynamic features such as velocity, it is included because of the difficulty that a potential forger has in accurately simulating the pressure profile. Mean pen-tip pressure used in this feature is simply the average of all values in the pressure profile for the stroke.

Standard Deviation of Pen-Tip Pressure: This gauges how much a writer typically varies his or her pen-tip pressure during the course of the stroke. Another purely dynamic feature, this is calculated as the regular standard deviation of the pressure profile.

Maximum Pen-Tip Pressure: This is the highest value in the pressure profile. Like all of the pressure features, the maximum pressure is not able to be extracted from an off-line copy of the writing and, while not as repeatable as velocity, is still useful in combination with other features.

Minimum Pen-Tip Pressure: This refers to the minimum non-zero value in the pressure profile. This is another personalised, invisible feature that previous researchers have also found useful [4].

Gradient of the Start and End Points: These two features capture the angle/gradient at the beginning and the end of the stroke. These values are calculated using the first two points and last two points of the stroke respectively. (See Figure 6.)

Gradient of Intermediate Tangents: The tangent of the stroke is obtained at three intermediate points equidistantly spaced within the stroke. These gradients (along with those in the previous feature) try to capture some element of the shape of the stroke and the direction changes within it.

This complete set of features was used in the initial experimentation in building the HSV system, but it became evident through further experimentation and examination of the interand intra-writer variability that some of these features were

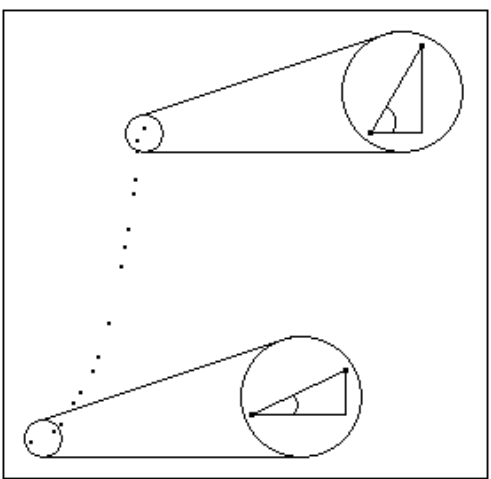

Fig. 6. A graphical representation of the beginning and ending gradient values within the stroke.

not insightful and the overall performance (in terms of both execution speed and classification error) suffered as a result of their inclusion. The removed features were as follows:

All acceleration and deceleration measurements: although these were initially thought to be useful (and are useful when taken over the signature as a whole), the values calculated using only the data points in the signature segment were not stable enough to be usable. There are not enough points in an average stroke to get a useful measure.

Standard Deviation of Pen-Tip Pressure: This feature simply lacked the stability to have any use in the HMM-based system and was removed from the feature set.

Horizontal Velocity: there were typically not enough points dispersed in the horizontal direction to get use out of this feature.

Gradient of the Start and End Points: similar to the previous features it was found that this feature did not exhibit enough stability to be considered useful and was therefore removed from the feature set.

Handwriting Slant Using Stroke End Points: the instability of this feature also rendered it unusable.

This leaves a total of fourteen discriminative features in the feature set used in the HMM.

\section{EXPERIMENTAL SETUP AND RESULTS}

This section describes the experimental setup used in the development and testing of the HMM-based HSV system, including a discussion of the different structures and setups used in an attempt to obtain optimum results. The signature database and pre-processing are described in McCabe et al [10]. The different sets of experiments performed are now considered in detail and grouped according to category.

1) Model Structure: Each individual's signature was modelled with a single HMM with the basic structure being a leftto-right (Bakis) model with self, forward and skip transitions between the states. The number of states in the model depends on the number of observations, a point further discussed below. The initial state probabilities and state transition matrix for each HMM is estimated during the training phase. The observation probabilities density function is approximated by 
a discrete distribution of vector quantiser indices. A discrete implementation was chosen over a continuous modelling approach using Gaussian mixtures partly because discrete models cope very well with the features typically used in handwriting [15]. Additionally, a vector quantiser can be derived during training for each individual signer, adding another level of personalisation to the HSV system. Finally, use of discrete distributions alleviates the need to make assumptions about the form of the underlying distribution.

2) Number of States: The selection of the most appropriate number of states to use in a HMM is not a trivial one. There is no heuristic approach to deciding on the number of states to use and the approach of other HSV researchers in the literature has been more one of trial-and-error and experience.

Other researchers used values of 0.75 or 0.8 times the number of observations to determine the number of model states [4], [5]. Experimentation with this HSV system included searches of constants $(5,10,20,50,100)$ and multiplication factors of 0.5 to 1.5 in increments of 0.05 . Ultimately the multiplication factor of 0.8 was found to perform slightly better than 0.75 or any other values. The factor of 0.8 is used as a basis for further experimentation presented in the paper.

3) Training Approaches: The approach to training the model is the next aspect in the developmental process to be considered. A single HMM was used to model the signature for each signer in the database. Training of the HMM was done using five randomly selected samples of the user's genuine signature. A conscious decision was made to attempt to minimise the number of genuine signatures to use in building the reference to minimise the inconvenience to a potential user. Many researchers use more than five reference signatures when training HMMs [6], [11], [19], which would be expected to produce better results (use of more reference signatures gives the HMM better insight into the signer's natural style), but the extra overhead and inconvenience to users may make the system less attractive.

The actual process of training the HMM involved experiments using both the Baum-Welch (BW) and Segmental K-Means (SKM) algorithms described above. Each takes a different approach to the training process and results in different training characteristics. Table I summarises the training performance details in terms of the number of iterations and the overall time required for the algorithm to converge. As can be seen from the table, the SKM approach converged, on average, in far fewer iterations than the BW approach (SKM actually converged in fewer epochs for every signer in the database). In addition it can be seen that SKM takes far less time to build the model than does the BW approach. Irrespective of the training approach used, the end result of the training process was a single trained HMM for each signer. It is then possible to determine the likelihood that a given test signature was generated by a particular signer's HMM.

For each remaining signature (both forgeries and genuine signatures not used in training the model) corresponding to the current signer, the forward algorithm (see McCabe et al [10]) was used to find the likelihood that the test signature was

\section{TABLE I}

The training performance of the two HMM training algorithms used in experimentation. The "Number of Epochs" is the mean number of epochs required for convergence to occur (with standard deviation in brackets). The relative time compares the elapsed time prior to convergence.

\begin{tabular}{|l|c|c|}
\hline Training Approach & Number of Epochs & Relative Time \\
\hline Baum-Welch & $236.0(138.5)$ & $100 \%$ \\
\hline Segmental K-Means & $5.2(1.4)$ & $2.1 \%$ \\
\hline
\end{tabular}

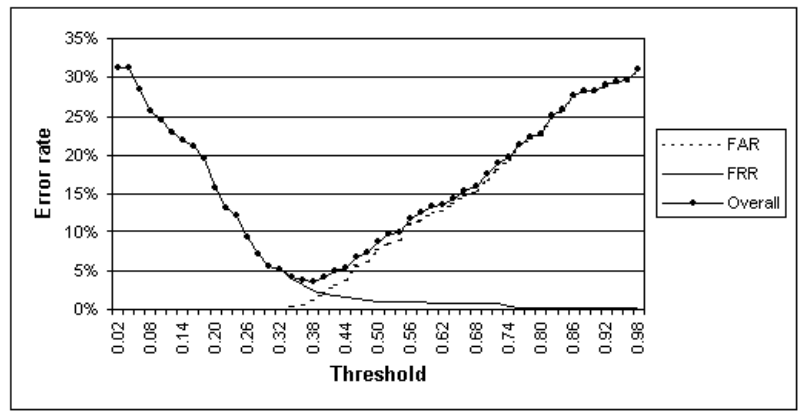

Fig. 7. A plot of the HSV results using the Segmental K-Means learning algorithm and different threshold values.

generated by the current signer's HMM (i.e., the likelihood that the test signature was produced by the same individual that performed the reference signatures). If this likelihood value was greater than an applied threshold value $T$, then the test signature was accepted as genuine, otherwise rejected as an attempted forgery. Error rates can be determined based on how many forgeries are accepted and how many genuine samples are rejected.

The performances of the two training algorithms are also compared in terms of the resulting error rates. Essentially this was measuring which of the two approaches best models the signer's natural writing style. Figures 7 and 8 present the error plots for the SKM and BW approaches respectively These show that in this instance the SKM approach more successfully models the signatures.

The significant error rates that measure how well the HSV performed (the error rates most often reported in the literature) for each learning approach appear in Table II. The values in this table more clearly shows the superior performance of the SKM approach that resulted in a 3.5\% OER compared to $5.0 \%$ for BW. Note that the value for zero-effort FAR (or ZEFAR, see McCabe et al [10] for more information on zero-effort forgeries) is calculated by using the genuine signatures of all other signers in the database as attempted forgeries. The threshold used in zero-effort FAR calculation is the same as that which produces the lowest OER.

There was some expectation that the two sets of error rates resulting from the two training approaches would differ. However, the magnitude of this difference was unexpected. Further experimentation using different sizes for the reference signature set reveals that the disparity is reduced when more reference signatures are used. Table III presents the error rates 


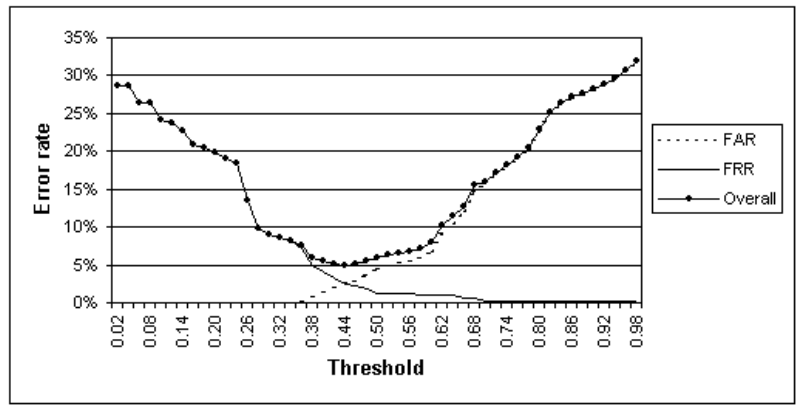

Fig. 8. A plot of the HSV results using the Baum-Welch learning algorithm and different threshold values.

TABLE II

A comparison of the modelling accuracy of the Segmental K-Means and Baum-Welch HMM training algorithms. All significant HSV-related error rates are reported in this table.

\begin{tabular}{|l|c|c|c|c|c|}
\hline Training Approach & FAR & FRR & OER & EER & ZEFAR \\
\hline Baum-Welch & $2.6 \%$ & $2.4 \%$ & $5.0 \%$ & $2.5 \%$ & $0.5 \%$ \\
\hline Segmental K-Means & $1.2 \%$ & $2.3 \%$ & $3.5 \%$ & $1.9 \%$ & $0.4 \%$ \\
\hline
\end{tabular}

resulting from the use of ten reference signatures to train the model. This table shows that the BW approach responded well to an increase in reference set size and doesn't seem to cope as well as SKM when this set size is small. This table also shows how dramatically the error rates can improve when more reference signatures are used. They may also be used to perform comparisons between this system and others developed using larger reference signature sets.

\section{CONCLUSIONS}

This paper presents a HSV system that is based on a HMM approach to representing and verifying the hand signature data. HMMs are naturally suited to modelling flowing entities such as signatures and speech. The HSV system described in this paper takes into account historical work performed in biometric verification using HMMs. It extracts several local features (both static and dynamic) from the signature data and models these using the best aspects of historical HMM modelling for HSV. The resulting system is tested on an experimental dataset that contains multiple samples of handwritten signatures (including both genuine and forgeries). The resulting HSV system performs reasonably well with an OER of $3.5 \%$ being reported in the best case experimental analysis.

As the NN approach is based on the analysis of global

TABLE III

A comparison of the modelling accuracy of the Segmental K-Means and Baum-Welch HMM training algorithms when ten reference signatures are used to train the model.

\begin{tabular}{|l|c|c|c|c|c|}
\hline Training Approach & FAR & FRR & OER & EER & ZEFAR \\
\hline Baum-Welch & $1.3 \%$ & $1.9 \%$ & $3.2 \%$ & $1.7 \%$ & $0.2 \%$ \\
\hline Segmental K-Means & $1.0 \%$ & $1.7 \%$ & $2.7 \%$ & $1.4 \%$ & $0.1 \%$ \\
\hline
\end{tabular}

features and the HMM model examines local aspects, future work involves combining both approaches to create a hybrid system. The results of both models can be combined to address the problems inherent in either approach alone in an attempt to further reduce the OER.

\section{REFERENCES}

[1] J.K. Baker. The DRAGON System - an Overview. IEEE Transactions on Acoustics, Speech and Signal Processing, Vol. 23, pp 24-29, 1975.

[2] J. Cai and Z-Q. Liu. Intergration of Structural and Statistical Information for Unconstrained Handwritten Numeral Recognition. IEEE Transactions on Pattern Analysis and Machine Intelligence, 21(3), pp 263-270, 1999.

[3] W. Cho, S. Lee and J.H. Kim. Modeling and Recognition of Cursive Words With Hidden Markov Models. Pattern Recognition, Vol. 28, No. 12, pp 1941-1953, 1995.

[4] J.G.A. Dolfing, E.H.L. Aarts and J.J.G.M. van Oosterhout. On-Line Signature Verification with Hidden Markov Models. Proceedings of the 14th International Conference on Pattern Recognition, Brisbane, Australia, pp 1309-1312, 1998.

[5] J.G.A. Dolfing and A. Wendemuth. Combination of Confidence Measures in Isolated Word Recognition. Proceedings of the International Conference on Spoken Language Processing, pp 3237-3240, 1998.

[6] R.S. Kashi, J. Hu, W.L. Nelson and W. Turin. On-line HSV using Hidden Markov Model Features. Proceedings of International Conference on Document Analysis and Recognition, 1997.

[7] K. Lee and H. Hon. Speaker-Independent Phone Recognition Using Hidden Markov Models. Transactions on Acoustics, Speech and Signal Processing, Vol. 37, No. 11, pp 1641-1648, 1989.

[8] A. McCabe. Markov Modelling of Simple Directional Features for Effective and Efficient Handwriting Verification. R. Mizoguchi and J. Slaney (Eds.): Pacific Rim International Conference of Artificial Intelligence (PRICAI) 2000, LNAI 1886, p 801, 2000.

[9] A. McCabe and J. Trevathan. A New Approach to Avoiding the Local Extrema Trap. In the Journal of the Australian and New Zealand Industrial and Applied Mathematics - ANZIAM J, vol. 48, C474-C493, ISSN 1446-8735, October 2007.

[10] A. McCabe, J. Trevathan and W. Read Neural Network-based Handwritten Signature Verification. Journal of Computers, vol. 8, Issue 3, 2008 (to appear).

[11] M.E. Munich. Applications of Hidden Markov Models to Signature Verification. Sensory Information Processing Laboratory, California Institute of Technology, Pasadena, California, 1998.

[12] R. Plamondon and M. Parizeau. Signature Verification from Position, Velocity and Acceleration Signals: A Comparative Study. Proceedings of 9th International Conference on Pattern Recognition, Vol. 1, 260-265, 1988.

[13] L. Rabiner and B. Juang. Fundamentals of Speech Recognition. Prentice Hall, New Jersey, 1993.

[14] G. Rigoll. and A. Kosmala. A Systematic Comparison between Online and Off-line Methods for Signature Verification. Proceedings of the International Conference on Pattern Recognition, Vol. II, pp 1755-1757, 1998.

[15] G. Rigoll, A. Kosmala, J. Rottland and C. Neukirchen. A Comparison between Continuous and Discrete Density Hidden Markov Models for Cursive Handwriting Recognition. Proceedings IEEE International Conference of Pattern Recognition, Vienna, Vol. II, pp 205-209, 1996.

[16] S.R. Veltman and R. Prasad. Hidden Markov Models Applied to Online Handwritten Isolated Character Recognition. IEEE Transactions on Image Processing, Vol. 3, No. 3, pp 314-318, 1994.

[17] A.J. Viterbi. Error Bounds for Convolutional Codes and an Asymptotically Optimal Decoding Algorithm. IEEE Transactions on Information Theory, IT-13, pp 260-269, 1967.

[18] L. Yang, B. Widjaja and R. Prasad. Applications of Hidden Markov Models for Signature Verification. Pattern Recognition, Vol. 28, No. 2, pp 161-170, 1995.

[19] H.S. Yoon, J.Y. Lee and H.S. Yang. An On-line Signature Verification System Using Hidden Markov Models In Polar Space. Proceedings of the Eighth International Workshop on Frontiers in Handwriting Recognition (IWFHR '02), 2002. 